\title{
MODELLING OF NONUNIFORM RC STRUCTURES FOR COMPUTER AIDED DESIGN
}

\author{
MUHAMMAD TAHER ABUELMA'ATTI \\ King Fahd University of Petroleum and Minerals, Box 203, Dhahran 31261, Saudi Arabia
}

(Received June 25, 1993; in final form August 28, 1993)

\begin{abstract}
A simple model for nonuniform distributed RC structures is presented. The model consists of three passive elements only and can be used for modelling nonuniform distributed RC structures involving exponential, hyperbolic sine squared, hyperbolic cosine squared and square taper geometries. The model can be easily implemented for computer-aided analysis and design of circuits and systems comprising nonuniform distributed RC structures.
\end{abstract}

\section{INTRODUCTION}

Nonuniform distributed parameter RC structures are widely used in many diverse fields; for example in integrated circuits, thin films as well as monolithics. This is attributed to the flexibility in tailoring and shaping their frequency response and time domain characteristics by exploiting their variable taper-geometry. For arbitrary taper-geometries, there is no general closed-form analytical solution for the transfer functions of these structures. Closed-form analytical expressions are available only for a few particular taper-geometries involving exponential, hyperbolic sine squared, hyperbolic cosine squared and square functions ${ }^{1}$. The use of these expressions, however, poses a formidable problem and involves considerable mathematical intricacy and, therefore, can not be used for obtaining the time-domain characteristics of these nonuniform RC structures. This is attributed to the difficulty in calculating the pole values of their transfer functions. Recourse to approximate models for the transfer functions of nonuniform distributed tapered RC structures is, therefore, inevitable. In this regard, Ghausi and Kelly ${ }^{1}$ used the m-pole and dominant-pole and excess-phase approximation methods for obtaining the unitstep response of exponentially tapered RC structures. Although, in principle, these methods can be used for obtaining the unit-step response of other taper-geometries, Ghausi and Kelly did not present such results; probably because of the rather tedious mathematical manipulations required to obtain the m-pole approximation and consequently the dominant-pole and excess-phase shift approximation.

In this paper, a general procedure will be presented for obtaining approximate closed-form analytical expressions for the transfer functions of the nonuniform distributed parameter RC structures with exponential, hyperbolic sine squared, hyperbolic cosine squared, and square taper-geometries. Using these expressions the unit-step response of these nonuniform distributed $\mathrm{RC}$ structures can be easily 
predicted. Moreover, using these expressions a simple model for these structures can be derived. Such model using three passive elements only, can be easily implemented for computer-aided analysis and design of circuits and systems comprising nonuniform distributed RC structures with exponential, hyperbolic sine squared, hyperbolic cosine squared and square taper geometries.

\section{ANALYSIS}

It is well known that the open-circuit voltage transfer function of two-port networks is given by

$T_{v}=\frac{-y_{21}(s)}{y_{22}(s)}$

The short-circuit admittance parameters $y_{21}(s)$ and $y_{22}(s)$ of the nonuniform distributed RC structures with exponential, hyperbolic sine squared, hyperbolic cosine squared, and square taper geometries may be written as follows [[1], eqn (4.2) of [2]],

$\mathrm{y}_{21}=-\Theta \operatorname{cosech} \theta \cdot[\mathrm{P}(0) \cdot \mathrm{P}(\mathrm{d})]^{1 / 2} / \mathrm{Z}_{0}$

$\mathrm{y}_{22}=\theta \operatorname{coth} \Theta \cdot P(d) / Z_{0}-P^{\prime}(d) \cdot d / 2 Z_{0}$

where $O=\left(B^{2}+Z_{0} Y_{0}\right)^{1 / 2}, B=k d, Z_{0}=r_{0} d, Y_{0}=s c_{0} d, k$ is a constant equal to the taper factor and $\mathrm{d}$ is the length of tapered $\mathrm{RC}$ structure. The prime denotes differentiation with respect to the distance variable $\mathrm{x}$ along the tapered structure. $P(0)$ and $P(d)$ are the terminal values of the electrical taper function $P(x)$ of the tapered RC structure. The value of $\mathrm{P}(\mathrm{x})$ for various kinds of taper are listed in Table I. $r_{0}$ and $c_{0}$ are the resistance and capacitance per unit length. Using eqns $(1-3)$ it is easy to show that the transfer function of the special taper-geometries under consideration can be written as follows

$\mathrm{T}_{\mathrm{v}}=\frac{\alpha}{\cosh \theta+\beta \frac{\sinh \theta}{\theta}}=\frac{1}{\mathrm{Q}(\psi)}$

where $\psi=\operatorname{sc}_{0} \mathrm{r}_{0} \mathrm{~d}^{2}$. The values of $\alpha, \beta$ and $\theta$ for various kinds of taper-geometry are listed in Table I. Fig. (1) shows plots of $\mathrm{Q}(\psi)$ for different values of kd and $\mathrm{kc}_{1}$ for the special cases of tapered $\mathrm{RC}$ structures under consideration.

Inspection of Fig. (1) reveals that, in general, $\mathrm{Q}(\psi)$ can be represented by polynomials of the Nth order of the form

$$
\mathrm{Q}(\psi)=1+\sum_{\mathrm{n}=1}^{\mathrm{N}} \gamma_{\mathrm{n}} \psi^{\mathrm{n}}
$$


NONUNIFORM RC STRUCTURES

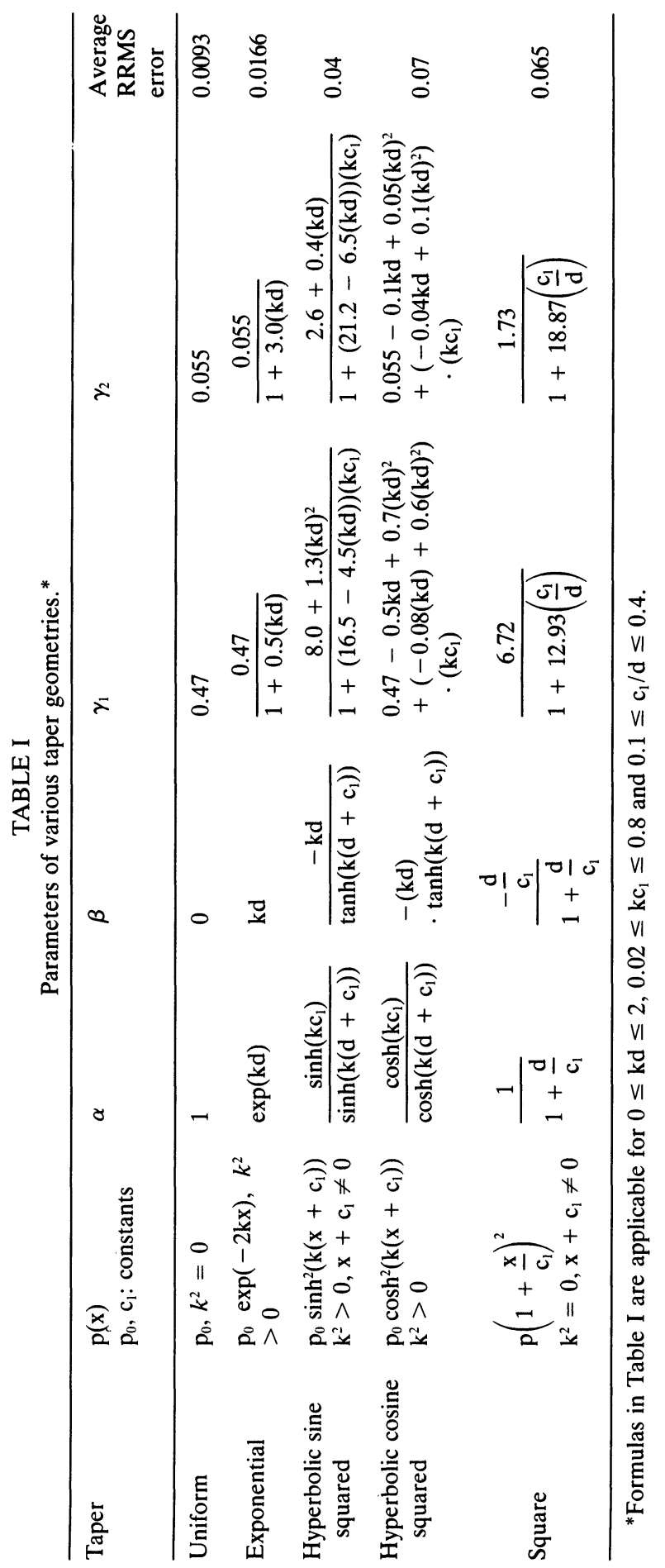




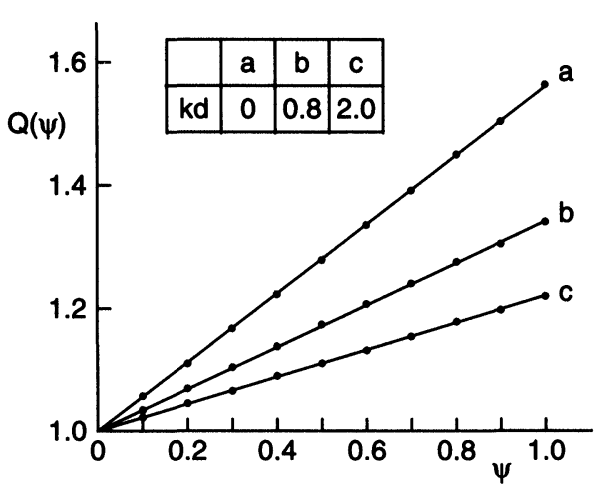

(a) Exponential taper. Curve (a) with $\mathbf{k d}=0$ represents uniform taper

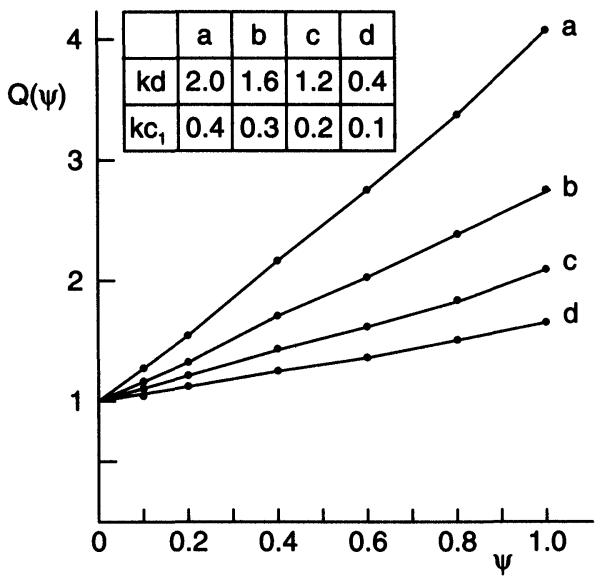

(c) Hyperbolic cosine squared

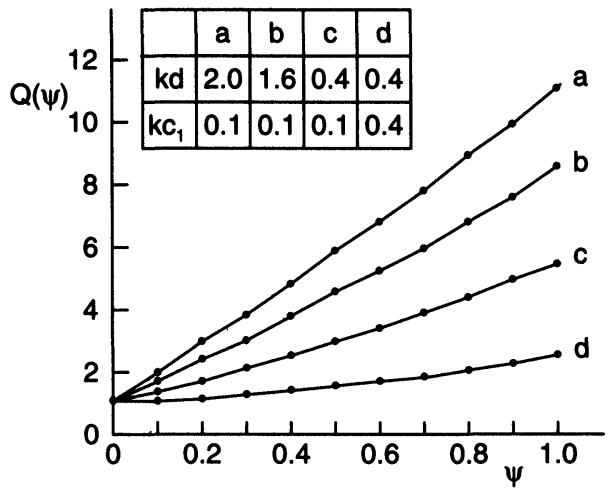

(b) Hyperbolic sine squared

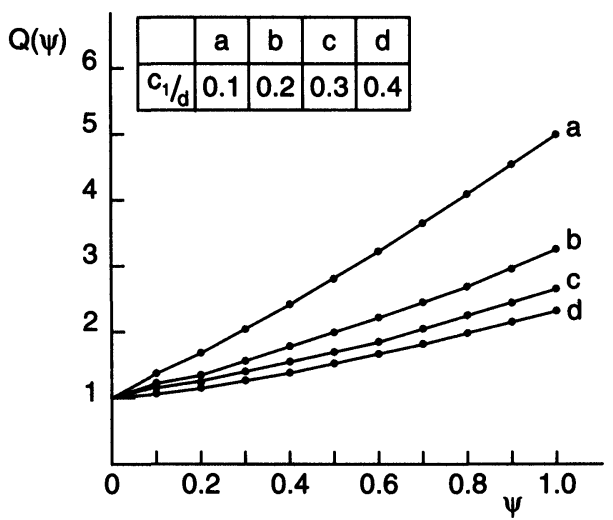

(d) Square taper

FIGURE 1 Variation of $\mathrm{Q}(\psi)$ with $\psi$ for different taper geometry, - calculated, eqn (4) .Calculated, eqn (5)

In general, the parameters $\gamma_{\mathrm{n}}$ can be obtained, for different values of $\mathrm{kd}$ and $\mathrm{kc}_{1}$, by using standard curve-fitting subroutines available in most mainframe computers. However, for second-order polynomials, with $N=2$, the parameters $\gamma_{1}$ and $\gamma_{2}$ can be obtained by hand calculation using the Lagrange interpolating polynomial ${ }^{3}$. This procedure yields a family of parameters $\gamma_{1}$ and $\gamma_{2}$ that depend on $k d$ and $\mathrm{kc}_{1}$. These parameters are fitted to simple closed-form analytical expressions. These expressions are listed in Table $\mathrm{I}$.

Using eqns $(4,5)$ calculations are performed, using $\mathrm{N}=2$, and are shown in Fig. (1) from which it is obvious that the proposed second-order polynomial of eqn. (5) accurately represents the function $\mathrm{Q}(\psi)$. The average relative-root-meansquare (RRMS) errors are listed in Table I. 


\section{RESULTS}

By combining eqns $(4,5)$ the unit-step response due to input $v(t)=u(t)$ is thus

$\mathrm{V}_{0}(\mathrm{~s})=\frac{1}{\mathrm{~s}} \frac{1}{1+\gamma_{1} \psi+\gamma_{2} \psi^{2}}$

or

$\frac{\mathrm{V}_{0}(\psi)}{\mathrm{r}_{0} \mathrm{c}_{0} \mathrm{~d}^{2}}=\frac{1}{\gamma_{2}} \overline{\psi\left(\psi^{2}+2 \mathrm{~b} \psi+\mathrm{w}_{0}^{2}\right)}$

where $2 b=\gamma_{1} / \gamma_{2}$ and $w_{0}^{2}=1 / \gamma_{2}$. Since $w_{0}^{2}<b^{2}$, then the inverse Laplace transform of eqn (6) is obtained $\mathrm{as}^{4}$

$\mathrm{v}_{0}(\mathrm{t})=1-\frac{\mathrm{w}_{0}^{2}}{\lambda-\delta}\left[\frac{\mathrm{e}^{\delta t / \mathrm{r}_{0} \mathrm{c}_{0} \mathrm{~d}^{2}}}{\delta}-\frac{\mathrm{e}^{\lambda t / \mathrm{r}_{1} \mathrm{c}_{0} \mathrm{~d}^{2}}}{\lambda}\right]$

where $\delta$ and $\lambda$ are the roots of the equation

$\psi^{2}+2 b \psi+w_{0}^{2}=0$

Fig. (2) depicts the unit-step responses for some of the nonuniform distributed RC structures under consideration. From Fig. (2) it is obvious that the results obtained using the approximation of eqn (5) are in excellent agreement with the exact responses published previously ${ }^{1.5}$.

From eqns $(4,5)$ it is obvious that the approximation presented here to represent the transfer function of the open-circuit nonuniform distributed RC-structures under consideration correspond to the equivalent circuit shown in Fig. (3). In contrast with previously proposed models ${ }^{2.5 .6}$, which require a relatively large number of passive elements, the equivalent circuit proposed here consists only of three passive elements and can be used for modelling nonuniform RC-structures with tapergeometries involving exponential, hyperbolic sine-squared, hyperbolic cosinesquared, and square functions.

\section{CONCLUSIONS}

A second-order polynomial representation has been proposed for the transfer functions of open-circuit nonuniform RC-structures involving exponential, hyperbolic sine squared, hyperbolic cosine squared, and square taper-geometries. Based on this representation a lumped network with three passive elements only can be used for modelling the performance of the open-circuit transfer characteristics of these 


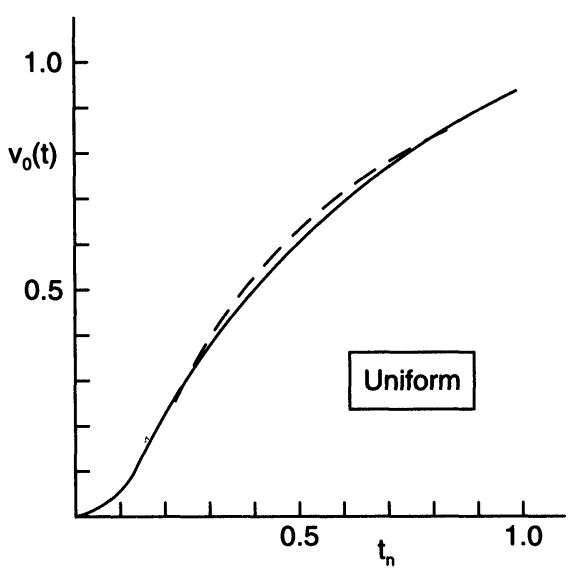

(a)

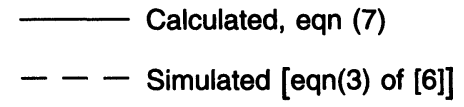

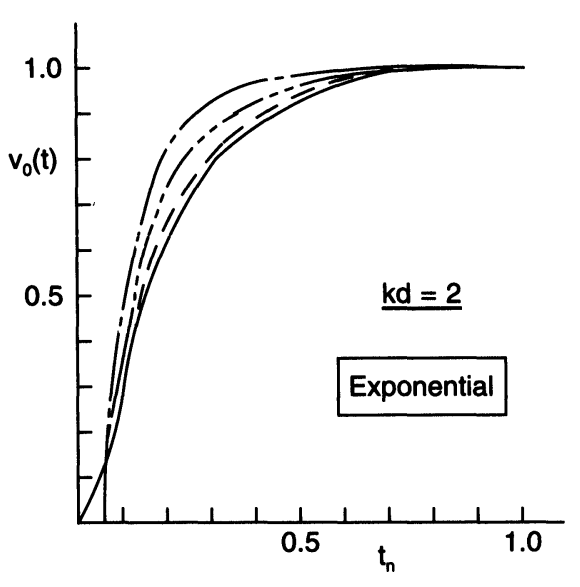

(b)

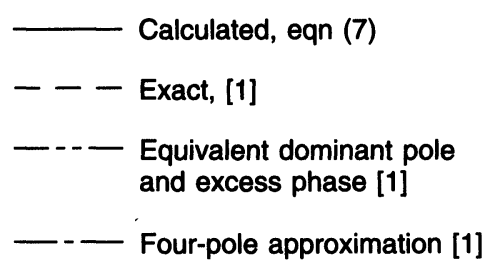

FIGURE 2 Unit step response of open-circuit uniform and exponential taper-geometries. $t_{n}=t / r_{0} c_{0} d^{2}$

nonuniform RC-structures. This model can be used for predicting the time-domain response of these structures. The proposed model can be easily implemented for computer-aided analysis and design of circuits and systems comprising exponential, hyperbolic sine squared, hyperbolic cosine squared, and square function nonuniform RC-structures.

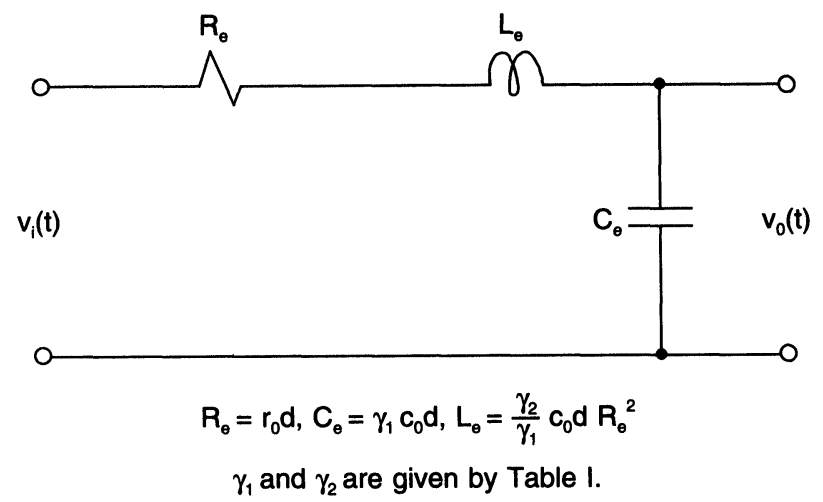

FIGURE 3 Proposed model for nonuniform RC structures involving exponential, hyperbolic sine squared, hyperbolic cosine squared, and square taper-geometries. 


\section{REFERENCES}

1. M.S. Ghausi and J.J. Kelly, Introduction to distributed-parameter networks with application to integrated circuits. Holt, Rinehart and Winston, New York, 1968.

2. U. Kumar and S.C. Dutta Roy, An accurate rational 2-port model of a class of tapered lossless and RC lines. International Journal of Circuit Theory and Applications, Vol. 5, 1977, pp. 355360).

3. R.L. Burden. J.D. Faires and A.C. Reynolds, Numerical analysis, PWS Publishers, Boston. MA, 1981.

4. L.A. Pipes and L.R. Harvill, Applied mathematics for engineers and physicists, McGraw-Hill, New York, 1970, pp. 769-771.

5. R.J. Antinone and G.W. Brown. The modelling of resistive interconnects for integrated circuits. IEEE J. Solid-State Circuits, Vol. SC-18, 1983, pp. 200-203.

6. G.D. Mey, A comment on the modeling of resistive interconnects for integrated circuits, IEEE J. Solid-State Circuits, Vol. SC-19, 1984, pp. 542-543. 

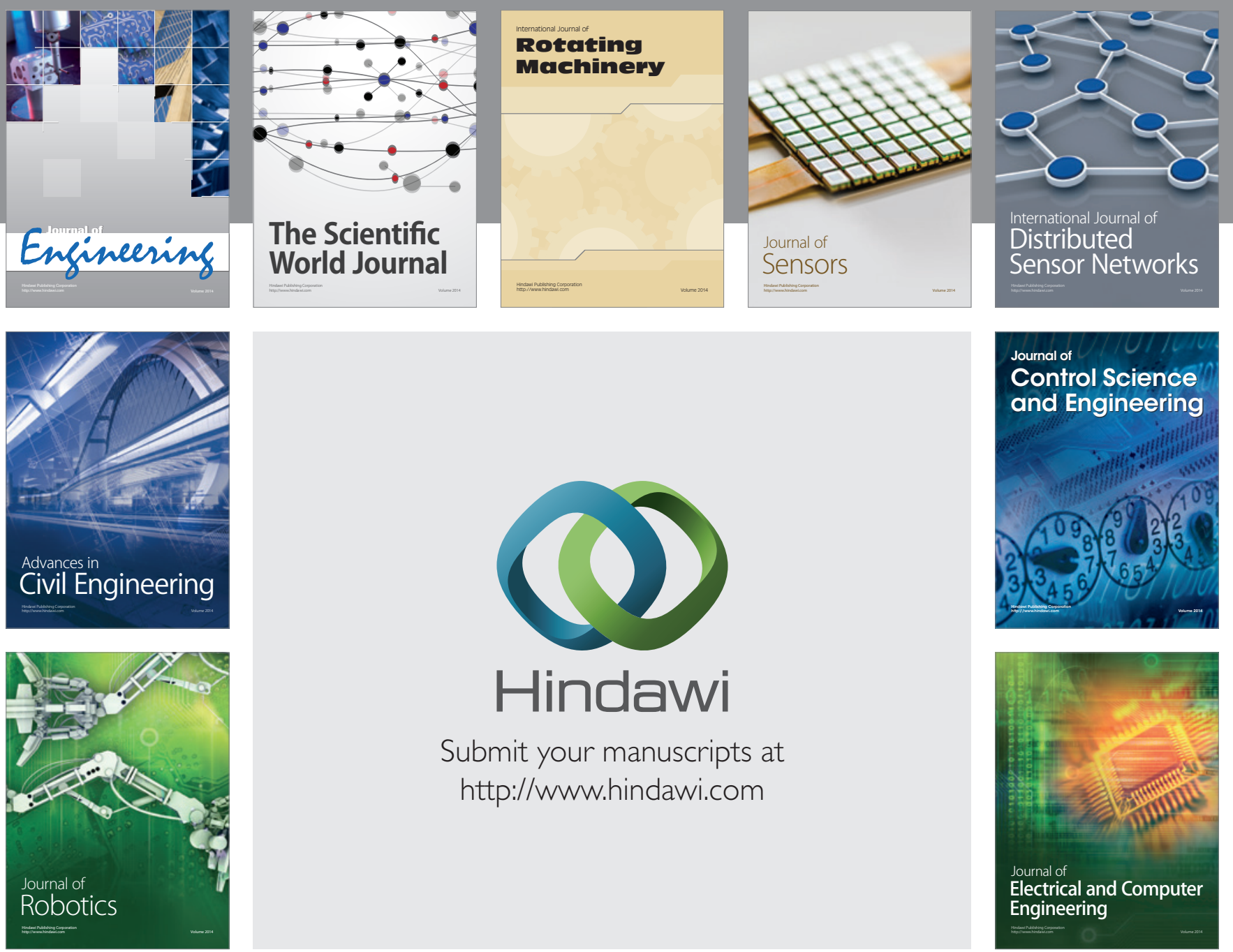

Submit your manuscripts at

http://www.hindawi.com
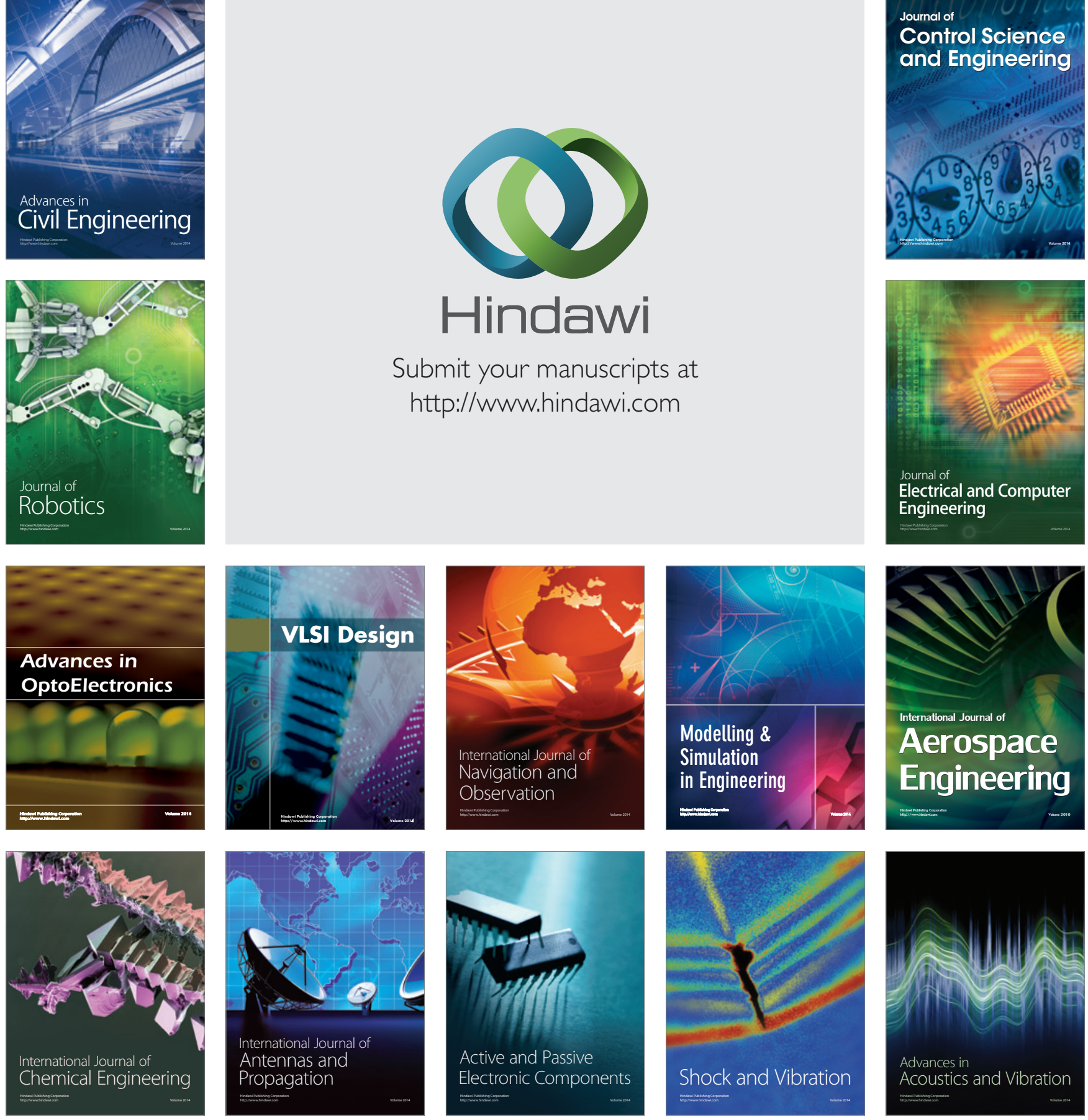